\title{
Resistencia a gentamicina, amikacina y ciprofloxacina en cepas hospitalarias de Klebsiella pneumoniae subespecie pneumoniae productoras de ß-lactamasas de espectro extendido
}

\author{
Patricia Díaz $\mathbf{Q}^{\mathbf{1 a}}$, Helia Bello $\mathrm{T}^{\mathbf{1 b}}$, Mariana Domínguez $\mathrm{Y}^{\mathbf{1 b}}$, \\ Natalia Trabal F1c, Sergio Mella $\mathbf{M}^{2}$, Raúl Zemelman $\mathrm{Z}^{3 \mathrm{~d}}$, \\ Gerardo González $\mathbf{R}^{1 \mathrm{e}}$. \\ Resistance to gentamicin, amikacin and \\ ciprofloxacin among nosocomial isolates of \\ Klebsiella pneumoniae subspecie pneumoniae \\ producing extended spectrum B-lactamases
}

Background: Klebsiella pneumoniae is a pathogenic bacterium frequently isolated from nosocomial samples, specially the subspecie pneumoniae, with extensive antibiotic resistance profiles, including third generation cephalosporins, aminoglycosides and quinolones. This is specially true for those strains producing extended spectrum beta lactamases (ESBL). Aim: To investigate the susceptibility to gentamicin, amikacin and ciprofloxacin and the presence of some aminoglycoside modifying enzyme (AMEs) among nosocomial strains of $\mathrm{K}$ pneumoniae subspecie pneumoniae producing ESBL Material and methods: The antibiotic resistant patterns and the level of resistance (minimal inhibitory concentration, MIC) of 100 strains, isolated from several hospitals of different Chilean cities, were determined. The presence of some aminoglycosides modifying enzyme (AMEs) was investigated by PCR. Results: Sixty five percent of strains were resistant to gentamicin, $47 \%$ were resistant to amikacin and $29 \%$ were resistant to ciprofloxacin. The most frequent AMEs genes detected were the aac (6')-Ib gene (6'$\mathrm{N}$-Acetyltransferase type Ib enzyme) in $69 \%$ of strains, conferring resistance to amikacin, kanamycin, tobramycin, and neomycin, and the gene aac(3)-IIa (3-Acetyltransferase type 3-IIa enzyme), in 36\% of strains, conferring resistance to gentamicin. Conclusions: Among nosocomial strains of $\mathrm{K}$ pneumoniae subspecie pneumoniae isolated from Chilean hospitals, there is an association between the production of ESBL and the resistance to others antimicrobial agents, especially aminoglycosides. Nevertheless, $71 \%$ of isolates are susceptible to ciprofloxacin (Rev Méd Chile 2004; 132: 1173-8).

(Key Words: Aminoglycosides; Antibiotic resistance, bacterial; Klebsiella pneumoniae)

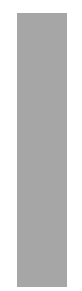

\footnotetext{
Recibido el 23 de marzo, 2004. Aceptado en versión corregida el 4 de agosto, 2004.

Financiamiento: Proyecto FONDECYT \#1020454.

${ }^{1}$ Departamento de Microbiología, Facultad Ciencias Biológicas, Universidad de Concepción. ${ }^{2}$ Departamento de Medicina Interna, Facultad de Medicina, Universidad de Concepción. ${ }^{3}$ Facultad de Ciencias y Tecnología, Universidad San Sebastián, Concepción, Chile.

aBioquímico. bBioquímico, Magíster en Microbiología. CLicenciada en Biología. dQuímico Farmacéutico. Master Science in Public Health. 'Licenciado en Biología, Magíster en Microbiología, Doctor en Ciencias Biológicas
}

Correspondencia a: Gerardo González R. Grupo de Investigación en Resistencia a Antibióticos (GIRA), Departamento de Microbiología, Facultad de Ciencias Biológicas, Universidad de Concepción, Concepción, Chile. Teléfono: 56-41-203237. Fax: 56-41-245975. E-mail: ggonzal@udec.cl 
$\mathrm{K}$ lebsiella pneumoniae, y en especial la subespecie pneumoniae, es un bacilo Gram negativo que se aísla frecuentemente de cuadros infecciosos del tracto respiratorio y vías urinarias, infecciones intraabdominales y bacteremias, principalmente en pacientes hospitalizados en unidades de cuidados intensivos y sometidos a terapia con antibióticos de amplio espectro ${ }^{1}$. Entre las enterobacterias, Klebsiella es el género que más se ha asociado con la producción de ß-lactamasas de espectro extendido (BLEE) y, a su vez, K pneumoniae subespecie pneumoniae ha llegado a ser la especie bacteriana en la cual se ha aislado y descrito el mayor número de estas enzimas ${ }^{2}$, por lo que se considera a este microorganismo como su principal reservorio a nivel hospitalario ${ }^{3}$. La importancia de las cepas hospitalarias de $\mathrm{K}$ pneumoniae productoras de BLEE radica en sus amplios patrones de resistencia a los antibió$\operatorname{ticos}^{4,5}$, que incluyen a cefalosporinas de tercera generación y, además, estas cepas con frecuencia poseen también resistencia a otros grupos de agentes antibacterianos de gran importancia clínica, como son los antibióticos aminoglicósidos y las quinolonas $^{6}$. Las BLEE han surgido como consecuencia de mutaciones puntuales sucesivas en los genes de ß-lactamasas de reducido espectro, como son las enzimas TEM-1, TEM-2, SHV-1 y OXA7, siendo su sustrato específico las penicilinas, las cefalosporinas (excepto cefamicinas) y los monobactámicos, ocasionando un problema terapéutico de notables dimensiones a nivel hospitalario.

Las BLEE clásicamente se han descrito como codificadas en elementos extracromosomales. Estos genes, y los que codifican la resistencia a otros antimicrobianos, pueden residir en el mismo plásmido conjugativo $\mathrm{y}$, por lo tanto, se transmiten juntos de un microorganismo a otro, confiriendo el perfil de resistencia antibiótica múltiple ${ }^{8}$.

También se han identificado estos genes en otros elementos genéticos, denominados integrones, que contienen un sistema de recombinación sitio específica que permite integrar y expresar genes de resistencia localizados en estructuras denominadas cassettes genéticos de resistencia ${ }^{9}$. Así, por ejemplo, se han informado genes que codifican ß-lactamasas que hidrolizan oxacilina y carbapenémicos $^{10,11}$. Los integrones también se pueden ubicar en plásmidos conjugativos, por lo que actualmente constituyen otra fuente de diseminación de genes de BLEE y de aquellos que codifican otras resistencias ${ }^{12}$.
Debido a que las cefalosporinas de tercera generación, junto a los antibióticos aminoglicósidos y las quinolonas constituyen las terapias antiinfecciosas de elección en el tratamiento de infecciones producidas por bacilos Gram negativos aeróbicos, el objetivo de este trabajo fue investigar la actividad de gentamicina, amikacina y ciprofloxacina; además de la presencia de genes de enzimas modificantes de antibióticos aminoglicósidos en cepas hospitalarias de K pneumoniae subespecie pneumoniae productoras de BLEE.

\section{MATERIAL Y MÉTODO}

Cepas bacterianas: En este estudio se incluyeron 100 cepas de $\mathrm{K}$ pneumoniae subespecie pneumoniae productoras de BLEE aisladas de muestras clínicas (25 de tracto respiratorio, 23 de orina, 17 de sangre, 7 de heridas, 6 de líquido ascítico y 22 de origen desconocido) en hospitales de diversas ciudades de Chile entre los años 1997 y 2003. La distribución de las cepas fue: 44 aisladas en hospitales de Santiago, 4 de Talca, 29 de Concepción, 15 de Talcahuano, 3 de Coronel, 3 de Lota y 2 de Puerto Montt. Las cepas se mantuvieron a $-70^{\circ} \mathrm{C}$, en una mezcla 2:1 de cultivo de $18-24 \mathrm{~h}$ y glicerol $50 \% \mathrm{v} / \mathrm{v}$.

Patrones y niveles de resistencia a gentamicina (GEN), amikacina (AMK), y ciprofloxacina (CIP): Estos parámetros fueron determinados por el método de difusión en agar y por dilución seriada en agar, respectivamente. Las recomendaciones y puntos de corte para definir resistencia bacteriana a los antibióticos ensayados fueron las indicadas por el National Committee for Clinical Laboratory Standards ${ }^{13,14}$. Se utilizaron como cepas controles Escherichia coli ATCC 25922 y Staphylococcus aureus ATCC 25923.

Detección de genes de enzimas modificantes de aminoglicósidos (EMA): Se realizó por RPC, utilizando partidores específicos para cada gen (Tabla 1) ${ }^{15-}$ 17. Se pesquisó los genes de las EMA más frecuentemente encontrados en cepas de enterobacterias $^{18}$ : aac(3)-IIa, acc(6')-Ib, ant(2")-Ia, ant(3")-Ia y aph(3')Ia. La RPC se realizó utilizando $2,5 \mu \mathrm{L}$ de una mezcla de dNTPs 10x (1,25 mM de cada uno: dATP, dCTP, dGTP y dTTP), 1,5 $\mu \mathrm{L}$ de cada partidor (25 pM), 2,5 $\mu \mathrm{L}$ de tampón de RPC 10x, 1,25 $\mu \mathrm{L}$ de $\mathrm{MgCl}^{2}(50 \mathrm{mM}), 5,6 \mu \mathrm{L}$ de agua destilada estéril (SDW), 0,15 $\mu \mathrm{L}$ de Taq DNA polymerasa $(5 \mathrm{U} / \mu \mathrm{L}) \mathrm{y}$ 

Tabla 1. Partidores específicos utilizados en la pesquisa de genes de enzimas modificantes
de antibióticos aminoglicósidos

\begin{tabular}{|c|c|c|c|}
\hline Gen & Partidores & $\begin{array}{l}\text { Secuencia nucleotídica } \\
5^{\prime} \rightarrow 3^{\prime}\end{array}$ & Referencia \\
\hline aac(3)-IIa & $\begin{array}{l}\text { aacC2 } \mathrm{F} \\
\text { aacC2 } \mathrm{R}\end{array}$ & $\begin{array}{l}\text { CGCTAAACTCCGTTACC } \\
\text { TAGCACTGAGCAAAGCC }\end{array}$ & 15 \\
\hline aac( $\left.6^{\prime}\right)-\mathrm{Ib}$ & $\begin{array}{l}\left.\text { aac( } 6^{\prime}\right)-\mathrm{Ib} F \\
\operatorname{aac}\left(6^{\prime}\right)-\mathrm{Ib} R\end{array}$ & $\begin{array}{l}\text { TATGAGTGGCTAAATCGAT } \\
\text { CCCGCTTTCTCGTAGCA }\end{array}$ & 16 \\
\hline ant(2")-Ia & $\begin{array}{l}\operatorname{aadB} F \\
\operatorname{aadB} R\end{array}$ & $\begin{array}{l}\text { CGTCATGGAGGAGTTGGACT } \\
\text { CGCAAGACCTCAACCTTTTC }\end{array}$ & Este trabajo \\
\hline ant(3")-Ia & $\begin{array}{l}\operatorname{aadA} F \\
\operatorname{aadA} R\end{array}$ & $\begin{array}{l}\text { ATGAGGGAAGCGGTGATCGCC } \\
\text { TCTTCCAACTGATCTGCGCGC }\end{array}$ & 17 \\
\hline aph(3')-Ia & $\begin{array}{l}\text { aphA1 F } \\
\text { aphA1 R }\end{array}$ & $\begin{array}{l}\text { ATTCAACGGGAAACGTCTTG } \\
\text { AACAGGAATCGAATGCAACC }\end{array}$ & Este trabajo \\
\hline
\end{tabular}

$10 \mu \mathrm{L}$ del templado de ADN. El templado se preparó de acuerdo a lo descrito por Reyes y $\mathrm{col}^{19}$.

Los productos de RPC se sometieron a electroforesis en geles de agarosa $1 \%$ a $100 \mathrm{~V}$ en tampón TAE 0,5x (0,4 M Tris-HCl, 0,02 $\mathrm{M} \mathrm{Na}_{2}$ EDTA. $_{2} \mathrm{H}_{2} \mathrm{O}, 0,2 \mathrm{M}$ acetato de sodio, 1,02 $\mathrm{M}$ ácido acético), y se visualizaron en un transiluminador de luz UV, luego de ser teñidos con bromuro de etidio (0,5 mg/L). Las condiciones de la RPC fueron: un ciclo de $96^{\circ} \mathrm{C}$ por $30 \mathrm{~s}, 55^{\circ} \mathrm{C}$ por $1 \mathrm{~min}$ y $70^{\circ} \mathrm{C}$ por $3 \mathrm{~min}$, seguido de 25 ciclos de $96^{\circ} \mathrm{C}$ por $15 \mathrm{~s}, 55^{\circ} \mathrm{C}$ por $30 \mathrm{~s}$ y $70^{\circ} \mathrm{C}$ por 3 min y una extensión final a $70^{\circ} \mathrm{C}$ por $5 \mathrm{~min}$.

\section{RESULTADOS}

La mayoría de las cepas de K pneumoniae subespecie pneumoniae productoras de BLEE fue resistente a gentamicina (65\%), teniendo amikacina una mejor actividad. Sin embargo, el porcentaje de cepas resistentes a este último compuesto también fue alto, alcanzando a $47 \%$ de las cepas. En cambio, ciprofloxacina presentó una mayor actividad antibacteriana, manteniéndose más de $70 \%$ de las cepas susceptibles a este compuesto, finalmente todos los aislamientos fueron susceptibles a imipenem (datos no presentados). El nivel de resistencia a los compuestos antibacterianos, exhibidos por las cepas estudiadas, se presenta en la Tabla 2. Para los antibióticos aminoglicósidos se observó niveles de resistencia moderados, con valores que fluctuaron entre $\leq 2 \mu \mathrm{g} / \mathrm{ml}$ y $>1.024 \mu \mathrm{g} / \mathrm{ml}$, encontrándose que para gentamicina los valores de CMI fueron más elevados, con una $\mathrm{CM}_{50}$ de $64 \mu \mathrm{g} / \mathrm{ml}$ y $\mathrm{CM}_{90}$ de $512 \mu \mathrm{g} / \mathrm{ml}$; mientras que amikacina ejerció una mayor actividad antibacteriana con valores de $\mathrm{CMI}_{50}$ y $\mathrm{CM}_{90}$ de $16 \mu \mathrm{g} / \mathrm{ml}$ y de $64 \mu \mathrm{g} / \mathrm{ml}$, respectivamente. En cambio, el nivel de resistencia para ciprofloxacina fue más bajo, ya que su $\mathrm{CMI}_{50}$ fue $\leq 1 \mu \mathrm{g} / \mathrm{ml}$ y la $\mathrm{CM}_{90}$ sólo alcanzó los $2 \mu \mathrm{g} / \mathrm{ml}$.

Los genes de EMA detectados en las cepas estudiadas se muestran en la Figura 1. Los genes aac( $\left.6^{\prime}\right)$-Ib y ant(3")-Ia fueron los que se encontraron con mayor frecuencia, detectándose en $69 \%$ y $68 \%$ de las cepas, respectivamente. Con frecuencias menores se detectaron los genes aph(3')-Ia (55\%) y aac(3)-IIa (36\%). No se encontraron cepas que tuvieran el gen ant(2")-Ia.

\section{DisCUSIÓN}

En este trabajo se investigó la actividad de gentamicina, amikacina y ciprofloxacina, antibióticos de frecuente uso a nivel hospitalario ${ }^{20,21}$ sobre cepas hospitalarias de K pneumoniae subespecie pneumoniae productoras de BLEE.

Los aminoglicósidos estudiados demostraron tener menor actividad que ciprofloxacina, de hecho, la mayonáa de los aislamientos fue resistente a gentamici- 
Tabla 2. Actividad antibacteriana de gentamicina, amikacina y ciprofloxacina sobre cepas de K pneumoniae subespecie pneumoniae productoras de BLEE

\begin{tabular}{|lcccc|}
\hline Antibiótico & Rango CMI & $\mathrm{CMI}_{50}$ & $\mathrm{CMI}_{90}$ & \% de resistencia \\
\hline Gentamicina & $\leq 2->1.024$ & 64 & 512 & 65 \\
Amikacina & $\leq 2-128$ & 16 & 64 & 47 \\
Ciprofloxacina & $\leq 1-1.024$ & $\leq 1$ & 2 & 29 \\
\hline
\end{tabular}

${ }^{*} \mathrm{CMI}$ : Concentración Mínima Inhibitoria ( $\left.\mu \mathrm{g} / \mathrm{ml}\right) ; \mathrm{CMI}_{50}$ y $\mathrm{CM}_{90}$ : CMI que inhibe al 50\% y al 90\% de las cepas, respectivamente.

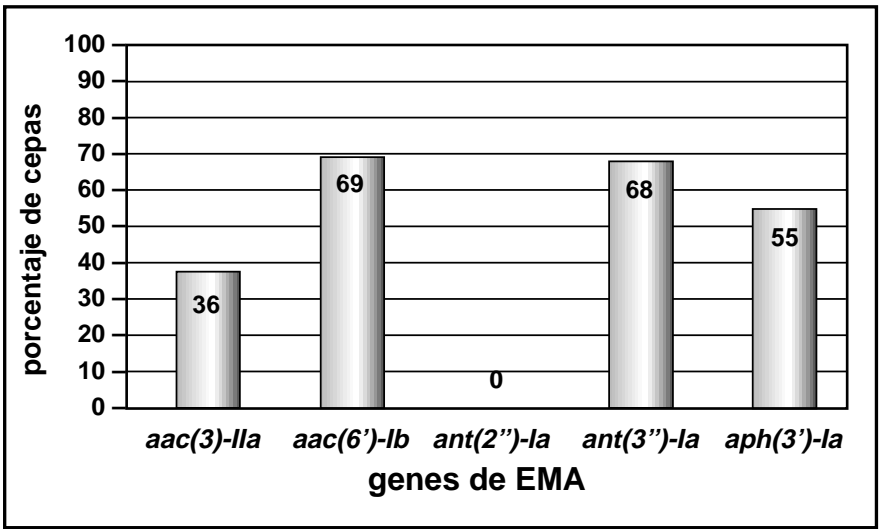

FiguRA 1. Distribución de genes de enzimas modificantes de aminoglicósidos (EMA) detectados en cepas de $\mathrm{K}$ pneumoniae subespecie pneumoniae productoras de BLEE, aisladas de diversos hospitales de Chile.

na. Amikacina demostró tener mayor actividad antimicrobiana que gentamicina, con valores de CMI que variaron entre $\leq 2 \mu \mathrm{g} / \mathrm{ml}$ y $128 \mu \mathrm{g} / \mathrm{ml}$, aun cuando casi el $50 \%$ de las cepas fue resistente a este compuesto. Un estudio realizado en Italia por Spanu y $\mathrm{col}^{6}$, describió esta diferencia de actividad, pero encontró frecuencias de cepas resistentes bastante menores a las obtenidas en este trabajo, además de valores de $\mathrm{CM}_{50}$ más bajos $(4 \mu \mathrm{g} / \mathrm{ml}$ y $2 \mu \mathrm{g} / \mathrm{ml}$ para amikacina y gentamicina, respectivamente). Estudios realizados en España ${ }^{22}$ y Corea 23 informaron situaciones similares.

Frente a ciprofloxacina, el comportamiento de las cepas fue diferente, ya que la mayoría de ellas continúa siendo susceptible a este antibiótico (71\%), con valores de $\mathrm{CMI}_{50}$ y $\mathrm{CM}_{90}$ de $\leq 1 \mu \mathrm{g} / \mathrm{ml}$ y $2 \mu \mathrm{g} / \mathrm{ml}$, respectivamente, lo cual concuerda con resultados descritos en la literatura ${ }^{24-26}$. No obstante, Spanu y $\operatorname{col}^{6}$ informaron un valor de $\mathrm{CM}_{90}$ más elevado (32 $\mu \mathrm{g} / \mathrm{ml})$. Sólo se observó una cepa con elevado nivel de resistencia a este compuesto (CMI $\geq 512 \mu \mathrm{g} / \mathrm{ml}$ ). La resistencia a ciprofloxacina, probablemente derive de mutaciones puntuales en los genes que codifican la $\mathrm{ADN}$ girasa o la topoisomerasa IV, aunque últimamente se ha descrito resistencia transferible en $\mathrm{K}$ pneumoniae $\mathrm{e}^{27,28}$.
La capacidad de las cepas de producir EMA se investigó determinando la presencia de los genes que las codifican y que, más frecuentemente, son detectados en las enterobacterias ${ }^{18}$. Se encontró que el gen aac(6')-Ib es el más frecuente en las cepas de $\mathrm{K}$ pneumoniae subespecie pneumoniae incluidas en este trabajo (69\%), seguido por el gen ant(3")-Ia (68\%); lo que concuerda con estudios previos ${ }^{19,26}$. La presencia de estos genes media la resistencia a amikacina, tobramicina, dibekacina, 2'-N-etilnetilmicina, netilmicina, 5-episisomicina, sisomicina, espectinomicina y estreptomicina ${ }^{18,29}$. Además, el gen aph(3')-Ia, tercero en frecuencia de detección (55\%), permite explicar la resistencia a kanamicina $\mathrm{y}$ neomicina que exhiben las cepas que lo tienen ${ }^{18,29}$. Por otra parte, el gen aac(3)-IIa, detectado en 36\% de las cepas, puede explicar en parte, la resistencia de este grupo de cepas a gentamicina, ya que la EMA por él codificada modifica a este antibiótico aminoglicósido, además de tobramicina, dibekacina, netilmicina y sisomicina ${ }^{29}$.

Es interesante destacar que dentro de las cepas que poseían el gen aac( $\left.6^{\prime}\right)-I b$, algunas de ellas presentaron susceptibilidad intermedia a amikacina y resistencia a gentamicina; en estas cepas, sin 
embargo no se encontraron genes que codificaran para EMA que modifiquen a gentamicina (aac(3)IIa, ant(2")-Ia). Esto podría deberse, por una parte, a lo ya informado por Casin y $\mathrm{col}^{30}$, quienes secuenciaron el gen aac(6')-Ib que tenían las cepas que presentaban estas características e informaron una mutación específica que da origen a esta alteración en el perfil de actividad de la enzima. Además, la resistencia a gentamicina puede explicarse por genes que no estuvieron incluidos en este estudio.

En este trabajo no se detectaron cepas de $\mathrm{K}$ pneumoniae subespecie pneumoniae que portaran el gen ant(2")-Ia, lo que concuerda con lo informado por Reyes y colli ${ }^{19}$ Interesantemente, White y $\mathrm{col}^{31}$ detectaron exclusivamente este gen como cassette genético de resistencia asociado a integrones en 7 cepas del género Klebsiella, y de éstas, 5 eran productoras de BLEE, indicando una asociación con este tipo de resistencia. En nuestro país, este gen también ha sido detectado en integrones, pero sólo en cepas de Escherichia coli ${ }^{19}$.

\section{REFERENCIAS}

1. Branger C, Lesimpie AL, Bruntau B, Berry P, LambertZeCHOVSKY N. Long-term investigation of the clonal disemination of Klebsiella pneumoniae isolates producing extended-spectrum ß-lactamases in a university hospital. J Med Microbiol 1998; 47: 201-9.

2. WEST PWJ. Extended-spectrum ß-lactamasesproducing Klebsiella spp. Br J Biomed Sci 2000; 57: 226-33.

3. RICE L. Evolution and clinical importance of extended-spectrum ß-lactamases. CHEST 2001; 119: 391-6.

4. Yang Y, Bhachech N, BradFord PA, Jett BD, Sahm DF, Bush K. Ceftazidime-resistant Klebsiella pneumoniae and Escherichia coli isolates producing TEM-10 and TEM-43 ß- lactamases from St. Louis, Missouri. Antimicrob Agents Chemother 1998; 42: 1671-6.

5. Zemelman R, Belo $H$, Domínguez M, González G, MeLia S, García A. Activity of imipenem, thirdgeneration cephalosporins, aztreonam and ciprofloxacin against multi-resistant Gram-negative bacilli isolated from Chilean hospitals. J Antimicrob Agents 1993; 32: 413-9.

6. Spanu T, Luzzaro F, Periu M, Amicosante G, Toniolo A, Fadda G and the Italian ESBL Study Group. Occurrence of extended-spectrum ß-lactamases in members of the family Enterobacteriaceae in Italy: Implications for resistance to
En los últimos años se ha observado un continuo aumento de aislamientos de enterobacterias, especialmente de $\mathrm{K}$ pneumoniae subespecie pneumoniae, productoras de BLEE y además resistentes a otras familias de antimicrobianos ${ }^{15,32}$. Nuestros resultados confirman que en nuestro medio hay una evidente asociación entre producción de BLEE y resistencia a quinolonas y aminoglicósidos-aminociclitoles.

Desde el punto de vista clínico, estos resultados explican las escasas alternativas terapéuticas distintas a los carbapenémicos para el tratamiento de las infecciones severas producidas por cepas productoras de BLEE. Sin embargo, dada la descripción cada vez mayor de resistencia de estos últimos agentes 33,34 , es práctico señalar, que al menos en determinados centros hospitalarios y en ciertas infecciones (tracto urinario) el uso de ciprofloxacina o amikacina, puede permitir, al menos en parte, disminuir el uso de carbapenémicos.

ß-lactams and other antimicrobial drugs. Antimicrob Agents Chemother 2002; 46: 196-202.

7. BRADFORD PA. Extended-spectrum ß-lactamases in the 21st century: Characterization, epidemiology, and detection of this important resistance threat. Clin Microbiol Rev 2001; 14: 933-51.

8. Bush K, Jacoby GA, Medeiros AA. A functional classification scheme for $ß$ - lactamases and correlation with molecular structure. Antimicrob Agents chemother 1995; 39: 1211-33.

9. BENNETT PM. Integrons and gene cassettes: a genetic construction kit for bacteria. J Antimicrob Chemother 1999; 43: 1-4.

10. NAAS T, NoRdmanN P. OXA-type- ß-lactamases. Curr Pharm Desig 1999; 5: 865-79.

11. Poirel L, Naas T, Nicolas D, Colet L, Bellais S, CAVALO JD ET AL. Characterization of VIM-2, a carbapenem-hydrolyzing metallo-ß-lactamase and its plasmid and integron borne gene from Pseudomonas aeruginosa clinical isolate in France. Antimicrob Agents Chemother 2000; 44: 891-7.

12. Martínez-Freijo P, Fluit AC, Schmitz FJ, Verhoef J, JoNES ME. Many class 1 integrons comprise distinct stable structures ocurring in different species of Enterobacteriaceae isolated from widespread geografic regions in Europe. Antimicrob Agents Chemother 1999; 43: 686-9.

13. National Committe For Cunical Laboratory Standards (NCCLS) 2002. Methods for dilution antimicrobial 
susceptibility tests for bacteria that grow aerobically. Approved standard. $5^{\text {th }}$ Edition. NCCLS document M7-A5. NCCLS, Wayne, Pennsylvania, USA.

14. National Committe For Clinical Laboratory StanDARDS (NCCLS) 2002. Performance standards for antimicrobial disk susceptibility tests approved standard. $7^{\text {th }}$ Edition. NCCLS document M2-A7. NCCLS, Wayne, Pennsylvania, USA.

15. Melano R, Corso A, Petroni A, Centrón D, Orman B, Pereyra A et al. Multiple antibiotic-resistance mechanisms including a novel combination of extended-spectrum ß-lactamases in a Klebsiella pneumoniae clinical strain isolated in Argentina. J Antimicrob Chemother 2003; 52: 36-42.

16. Senda K, Arakawa Y, Ichiyama S, NaKashima K, Ito $\mathrm{H}$, OHSUKa S ET al. PCR detection of metallo- $\beta$ lactamase gene $\left(\mathrm{bla}_{\mathrm{IMP}}\right)$ in Gram-negative rods resistant to broad-spectrum ß-lactams. J Clin Microbiol 1996; 34: 2904-13.

17. Mazel D, Dychinco B, Webb VA, Davies J. Antibiotic resistance in the ECOR collection: Integrons and identification of a novel aad gene. Antimicrob Agents Chemother 2000; 44: 1568-74.

18. VAKUlenko S, Mobashery S. Versatility of aminoglycosides and prospects for their future. Clin Microbiol Rev 2003; 16: 430-50.

19. Reyes A, Bello H, Dominguez M, Melia S, Zemelman $\mathrm{R}$, GonZÁlez G. Prevalence and types of class 1 integrons in aminoglycoside-resistant Enterobacteriaceae from several Chilean hospitals. J Antimicrob Chemother 2003; 51: 317-21.

20. Melia S, García A, Aguilera L, Zemelman R. Antibióticos aminoglicósidos. Agrupación según su estructura química y actualización sobre alguna de sus propiedades. Acta Microbiológica 1993; 4: 5-13.

21. Meuja S, acuña $G$, Muñoz M, Peréz C, Labarca J, GoNZÁLEZ G ET AL. Quinolonas: Aspectos generales sobre su estructura y clasificación. Rev Chil Infect 2000; 17: 53-66.

22. Ardanuy C, Lñ̃ares J, Domínguez MA, HernándezAués S, Benedí VJ, Martínez-Martínez L Outer membrane profiles of clonally related Klebsiella pneumoniae isolates from clinical samples and activities of cephalosporins and carbapenems. Antimicrob Agents Chemother 1998; 42: 1636-40.

23. Kim YK, Pai H, Lee HJ, Park SE, Choi EH, Kim J et AL. Bloodstream infections by extended-spectrum ß-lactamase-producing Escherichia coli and Klebsiella pneumoniae in children: epidemiology and clinical outcome. Antimicrob Agents Chemother 2002; 46: 1481-91.

24. Shannon K, Stapieton P, Xiang X, Johnson A, Beattie
H, EL BAKRI F ET AL. Extended-spectrum ß-lactamase producing Klebsiella pneumoniae strains causing nosocomial outbreaks of infection in the United Kingdom. J Clin Microbiol 1998; 36: 3105-10.

25. Siu LK, Lu PL, Hsueh PR, Lin FM, Chang S, Luh KT ET AL. Bacteremia due to extended-spectrum ßSlactamase-producing Escherichia coli and Klebsiella pneumoniae in a pediatric oncology ward: Clinical features and identification of different plasmids carrying both SHV- 5 and TEM- 1 genes. J Clin Microbiol 1999; 37: 4020-7.

26. Del Solar E, García A, Bello $\mathrm{H}$, Domínguez $\mathrm{M}$, GonZÁlez G, Zemelman R. Mecanismos enzimáticos de resistencia a antibióticos aminoglicósidos en bacilos Gram negativos de hospitales chilenos. Rev Méd Chile 1995; 123: 293-7.

27. Ruiz J. Mechanisms of resistance to quinolones: target alteration, decreased accumulation and DNA gyrase protection. J Antimicrob Chemother. 2003; 51: 1109-17.

28. Martínez-Martínez L, Pascual A, Jacoby GA. Quinolone resistance from a transferable plasmid. Lancet 1998; 351: 797-9.

29. Shaw KJ, Rather PN, Hare RS, Muler GH. Molecular genetics of aminoglycoside resistance genes and familial relationships of the aminoglycoside-modifying enzimes. Microbiol Rev 1993; 57: 138-63.

30. Casin I, Bordon F, Bertin P, Coutrot A, Podgiajen I, BRASSEUR R ET AL. Aminoglycoside 6'-N-acetiltransferase variants of the Ib type with altered substrate profile in clinical isolates of Enterobacter cloacae and Citrobacter freundii. Antimicrob Agents Chemother 1998; 42: 209-15.

31. White PA, Melver CJ, RawLinson WD. Integrons and gene cassettes in the Enterobacteriaceae. Antimicrob Agents Chemother 2001; 45: 2658-61.

32. RAHAL JJ. Extended-spectrum ß-lactamases: how big is the problem? Clin Microbiol Infect 2000; 6 (Suppl. 2): 2-6.

33. Mahgoub S, Ahmed J, Glatt AE. Completely resistant Acinetobacter baumannii strains. Infect Control Hosp Epidemiol 2002; 23: 477-9.

34. OLVER A. Resistencia a carbapenemas y Acinetobacter baumannii. Enferm Infecc Microbiol Clin 2004; 22: 259-61.

Agradecimientos:

Agradecemos a la señora Magda Hernández y al señor Andrés Soto, por su asistencia técnica, como también a todos los laboratorios de los hospitales incluidos en este estudio, por enviarnos desinteresadamente las cepas analizadas en este trabajo. 